\title{
Novel Conjugated Polymer Containing Anthracene Backbone: The Microstructure of Polymer Composed of 1,4-Benzenedithiol with 9,10-Diethynylanthracene and Isomerization of the Polymer and Model Compounds
}

\author{
Jian JiANG, Eiichi KoBAYASHI,* Sadahito AOSHIMA, \\ and Junji FURUKAWA \\ Department of Industrial Chemistry, Faculty of Science and Technology, \\ Science University of Tokyo, Noda, Chiba 278, Japan
}

(Received November 9, 1989)

\begin{abstract}
In order to determine the microstructure of the polymer composed of 1,4benzenedithiol with 9,10-diethynylanthracene (3), the cis and trans model compounds (1), (2) were prepared. In comparison with the characteristic absorption peaks of vinylene in the model compounds, the microstructure of the polymer was evaluated. Regardless of the initiation mode, UV irradiation, AIBN and none, the cis contents of the polymers were almost in the range of 80 to $85 \%$. The behavior of the isomerization of the polymer was also investigated by UV irradiation. The cis configuration of the polymer isomerized gradually to the trans one with increase of the UV irradiation time. The characteristic features of the isomerization were also studied using model compounds. The cis isomer of the model compound undergoes irreversible isomerization to the trans one by UV irradiation. In the presence of a radical source in the reaction system, both the cis and trans isomers can isomerize and reach an equilibrium state after reaction for a sufficient time. The isomerization mechanism was also discussed based on the potential energy diagram.

KEY WORDS Conjugated Polymer / Polymer Microstructure / 1,4-Benzenedithiol / 9,10-Diethynylanthracene / IR Spectra / Model Compound / Photoisomerization / Thermal Isomerization / Potential Energy Surface / Excitation State /
\end{abstract}

Recently, many kinds of conjugated polymers containing aromatic rings and a sulfur in the polymer backbone have been synthesized by the addition polymerization of dithiol derivatives to diethynyl derivatives. ${ }^{1-3}$ These polymers have attracted a great deal attention at present because of thier unusual electronic and optical properties. ${ }^{1,2}$ For example, the crystallinity of the polymer obtained in the addition polymerization of 1,4-benzenedithiol to 1,4-diethynylbenzene in solid state by UV irradiation could be reversibly changed by the irradiation of characteristic lights. The isomerization of the cis to trans configuration seems to induce decrease in the crystallinity of the polymer by a destruction of the semi-stable layer structure of the polymer. ${ }^{4}$

In this series, the authors reported the addition polymerizations of 1,4-benzenedithiol and 9,10-anthracenedithiol to 9,10-diethynylanthracene. ${ }^{5,6}$ These polymers containing anthracene rings in the polymer backbone exhibited high orientation and crystallinity caused by the accumulations or the aggregation of anthracene rings in the polymers. The anthracene unit in the polymer backbone provides an excellent conjugated $\pi$ system along the polymer chain that is necessary for the high

* To whom all correspondence should be addressed. 
nonlinear optical activity (molecular susceptibility). ${ }^{7,8}$ The relations between these physical properties and the microstructure of these anthracene containing polymers are under investigation at present.

This paper deals with the determination of the microstructure of the polymer composed of 1,4-benzenedithiol with 9,10-diethynylanthracene and the elucidation of the isomerization behavior of the polymer and model compounds.

\section{EXPERIMENTAL}

\section{Monomers}

1,4-Benzenedithiol (BDT) $)^{2}$ and 9,10-diethynylanthracene $(\mathrm{DEA})^{5}$ were synthesized according to the references. Thiophenol (Wako Pure Chemical Industries) was purified by distillation under reduced pressure before use.

\section{Addition Polymerization}

The addition polymerization of BDT to DEA was carried out in a pyrex tube in THF at $50{ }^{\circ} \mathrm{C}$ by UV irradiation, using high pressure mercury lamp 450W (Ushio Co.) under nitrogen atmosphere. The precipitated polymer as an insoluble polymer was recovered by a centrifugation, washed with THF several times and dried in vacuo at room temperature overnight. The soluble polymer in the residue was poured into a large excess of methanol and the precipitated polymer was recovered as a soluble polymer by a centrifugation, washed with methanol several times and dried in vacuo at room temperature overnight. These obtained polymers were weighed to calculate the yields and characterized.

\section{Preparation of the Model Compounds}

The cis and trans isomers of the model compounds, 9,10-bis(phenylthiovinylene)anthracene were prepared following the scheme shown below (Scheme 1).

9,10-Diethynylanthracene $\quad(0.34 \mathrm{~g}, \quad 0.0015$ $\mathrm{mol})$ and thiophenol $(0.33 \mathrm{~g}, 0.0030 \mathrm{~mol})$ in THF $(50 \mathrm{ml})$ were charged in a pyrex tube under nitrogen atmosphere, then, it was irradiated by UV light for $15 \mathrm{~min}$. The reaction proceeded almost quantitatively, and gave a mixed product containing all-cis isomer, cistrans isomer and benzenedisulfide. After the purification by an activated alumina column using equivolume mixture of hexane and benzene as an eluent, and then recrystallization in chloroform, a pure cis isomer (1) was obtained with about $34 \%$ yield. mp: $241-$ $242{ }^{\circ} \mathrm{C}$. Sulfur content: calcd. $14.4 \%$, found. $14.3 \%$. MS: $\mathrm{M}^{+}, 446 ; \mathrm{M}+1^{+}, 447 .{ }^{1} \mathrm{H}$ NMR (in $\mathrm{CDCl}_{3}$ at $25^{\circ} \mathrm{C}$ ), $\delta$ (ppm): 8.26 (q, anthracene ring, $2 \mathrm{H}), 7.54$ (q, anthracene ring, $2 \mathrm{H}$ ), $7.38-7.20(\mathrm{~m}$, benzene ring, $5 \mathrm{H}), 7.24(\mathrm{q}$, cis vinylene, $2 \mathrm{H}$ ); $J_{\text {cis }}, 10.01 \mathrm{~Hz}$. UV (in THF at $\left.20^{\circ} \mathrm{C}\right): \lambda_{\text {max }}, 401.8 \mathrm{~nm}\left(\varepsilon, 13 ; 5001 \mathrm{~mol}^{-1} \mathrm{~cm}^{-1}\right)$.

The trans isomer (2) could not be synthesized directly by the irradiation of UV light to the mixture of DEA and thiophenol. It was prepared by the isomerization of the cis isomer obtained by the above procedure. The trans isomer was also purified by the activated alumina column and the recrystallization in acetone before characterization. Yield: $32 \%$ mp:

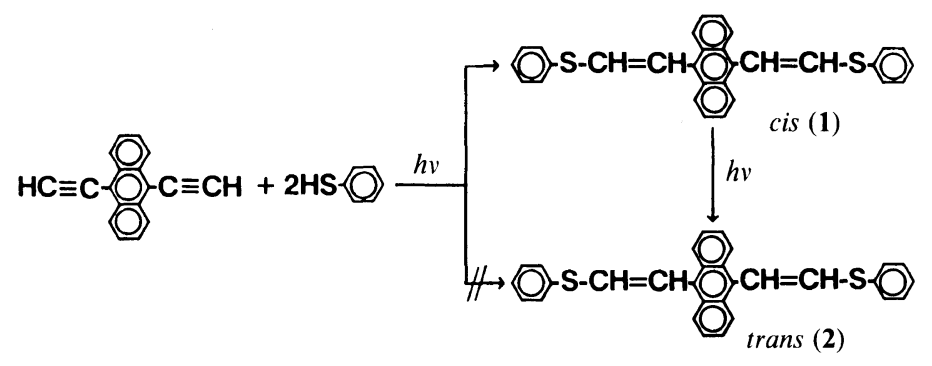

Scheme 1 . 
$192-193$ C. Sulfur content: calcd. $14.4 \%$, found. $14.4 \%$. MS: $\mathrm{M}^{+}, 446, \mathrm{M}+1^{+}, 447 .{ }^{1} \mathrm{H}$ NMR (in $\mathrm{CDCl}_{3}$ at $25^{\circ} \mathrm{C}$ ), $\delta$ (ppm); 8.32 (q, anthracene ring, $2 \mathrm{H}), 7.54-7.49(\mathrm{~m}$, anthracene and benzene rings, $5 \mathrm{H}), 7.38-7.26(\mathrm{~m}$, benzene ring, $2 \mathrm{H}), 7.09$ (q, trans vinylene, $2 \mathrm{H})$; $J_{\text {trans }}, 15.38 \mathrm{~Hz}$. UV (in THF at $20^{\circ} \mathrm{C}$ ): $\lambda_{\text {max }}$, $404.6 \mathrm{~nm}\left(\varepsilon, 13,4001 \mathrm{~mol}^{-1} \mathrm{~cm}^{-1}\right)$.

\section{Characterization}

The following instruments were used for the characterization of model compounds and polymers: ${ }^{1} \mathrm{H}$ NMR: JEOL PMX-60si, JEOL JNM FX-400; IR: Hitachi 260-50, FT-IR JEOL JIR-5300; UV: Shimadzu UV-260; Mass spectra: JMS D-300. The contents of sulfur in model compounds were determined by means of Schoeniger's method. ${ }^{1}$ The molecular weight of the polymers was measured by the method of end group determination, so called copper acetylide method. ${ }^{1}$

\section{RESULTS AND DISCUSSION}

Previously, the authors synthesized a novel conjugated polymer containing anthracene and sulfur in the polymer backbone (3) by the addition polymerization of BDT to DEA (Scheme 2). ${ }^{5}$

The obtained polymer dose not dissolve in any conventional organic solvents, so that infrared spectroscopic measurement by $\mathrm{KBr}$ disk method was mainly used to investigate the polymer structure. However, the configuration of the vinylene unit in the polymer backbone has been as yet unknown.

\section{Preparation of Model Compounds}

To determine the microstructure of the polymer (3), the authors used a comparative method of the IR spectra between the polymer and those of model geometrical isomers. These model compounds were prepared according to the Scheme 1: the cis isomer was synthesized by radical addition of thiophenol to DEA in THF; the trans one was obtained by the UVinduced isomerization of the cis one (Details see EXPERIMENTAL).

Figure 1 shows the IR spectra of the model compounds (cis and trans isomers) measured by $\mathrm{KBr}$ disk method. The characteristic absorption peaks of the vinylene out-of-plane deformation vibration were observed at $1320 \mathrm{~cm}^{-1}$ (cis isomer) and $945 \mathrm{~cm}^{-1}$ (trans isomer), respectively. This assignment of these characteristic vinylene peaks was undertaken in comparison with the cis and trans adducts prepared from the radical addition of thiophenol to phenylacetylene. ${ }^{9}$ The extinction coefficient (E) of the cis isomer at $1313 \mathrm{~cm}^{-1}$ is $98.41 \mathrm{~mol}^{-1} \mathrm{~cm}^{-1}$ and that of the trans one at $945 \mathrm{~cm}^{-1}$ is $130.71 \mathrm{~mol}^{-1} \mathrm{~cm}^{-1}$ in $\mathrm{CS}_{2}$ at $25^{\circ} \mathrm{C}$ measured by FT-IR (JEOL JIR-5300). The $\varepsilon$ of the cis and trans vinylene in the polymer cannot be determined in common organic solvents because of their insolubility. Accordingly, the cis and trans contents of the

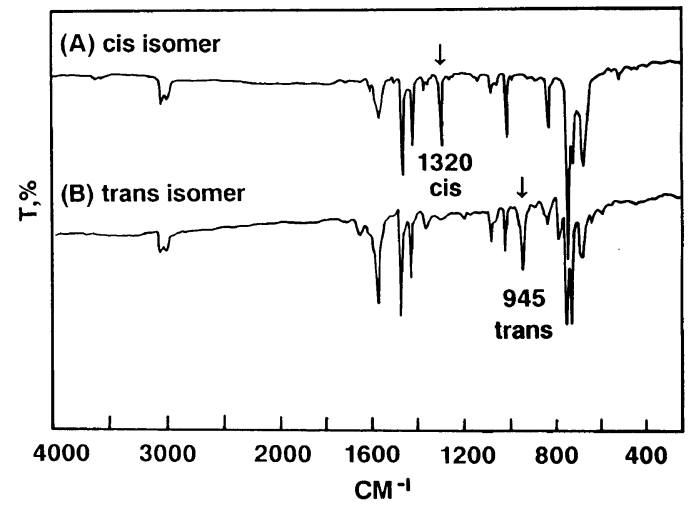

Figure 1. IR spectra of (A) cis isomer, (B) trans isomer of model compound, 9,10-bis(phenylthiovinylene)anthracene in $\mathrm{KBr}$ disk method.

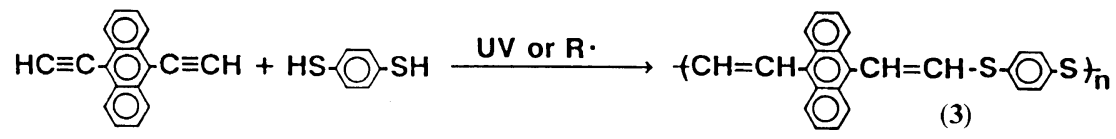

Scheme 2. 
vinylene in the polymer backbone were indirectly estimated with $\mathrm{KBr}$ disk method by using the $\varepsilon$ values of the cis and trans isomers of model compounds in $\mathrm{CS}_{2}$. The characteristic band of the cis vinylene at $1313 \mathrm{~cm}^{-1}$ measured in $\mathrm{CS}_{2}$ shifted to $1320 \mathrm{~cm}^{-1}$ by $\mathrm{KBr}$ disk method. The coefficients in the Morero ${ }^{10}$ type equations were calibrated with 7 samples comprising cis and trans isomers at various ratios as well as the errors are controlled in the order of $2 \%$. Then, the concentrations of the cis vinylene ( cis $\%$ ) and the trans one (trans $\%$ ) of the polymer backbone were determined by the following equations, where $D_{1320}$ and $D_{945}$ mean absorbances at 1320 and $945 \mathrm{~cm}^{-1}$, respectively.

$$
\begin{aligned}
& C=3.98 \times 10^{-3} D_{1320}-4.02 \times 10^{-4} D_{945} \\
& T=4.20 \times 10^{-3} D_{945}-2.43 \times 10^{-4} D_{1320} \\
& \operatorname{cis}(\%)=C /(C+T) \times 100 \\
& \operatorname{trans}(\%)=T /(C+T) \times 100
\end{aligned}
$$

\section{Microstructure of Polymers}

Figure 2 shows the IR spectrum of the polymer obtained by UV irradiation. The IR spectra of the other polymers synthesized in the presence of AIBN or without any initiator are the same as that obtained by UV irradiation. The characteristic absorption peaks of the cis and trans vinylene in the polymer backbone were observed at $1320 \mathrm{~cm}^{-1}$ and $945 \mathrm{~cm}^{-1}$. Using the above equations the microstructure of the polymer is estimated to be that the cis vinylene content is $85 \%$ and the trans one is $15 \%$.

Table I summarized the effect of the initiation mode on the microstructure of the polymers. The polymerization of BDT to DEA was carried out in tetrahydrofuran (THF) at $50^{\circ} \mathrm{C}$ under nitrogen atmosphere. Previous studies $^{5}$ on the polymer structure showed that the resulted polymer has an alternating structure composed of BDT with DEA. The yeild of the polymer obtained without initiator (Table I, No. 5) is relatively high, which would

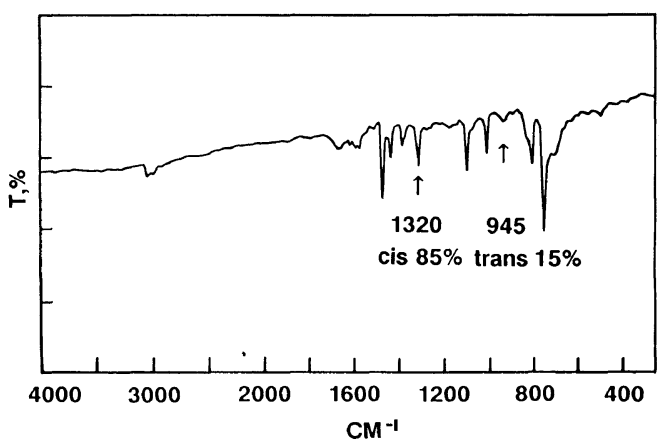

Figure 2. IR spectrum of the insoluble polymer composed of BDT with DEA in $\mathrm{KBr}$ disk method (Table I,

\begin{tabular}{|c|c|c|c|c|c|c|c|c|}
\hline \multirow{2}{*}{ No. } & \multirow{2}{*}{$\begin{array}{l}\text { Initiation } \\
\text { mode }^{\mathrm{b}}\end{array}$} & \multirow{2}{*}{$\begin{array}{c}\begin{array}{c}\text { Polymerization } \\
\text { time }\end{array} \\
\frac{\min }{}\end{array}$} & \multicolumn{2}{|c|}{ Yield $/ \%$} & \multicolumn{2}{|c|}{$\bar{M}_{n} \times 10^{-3 \mathrm{c}}$} & \multicolumn{2}{|c|}{ cis $\%$} \\
\hline & & & Insol. & Sol. & Insol. & Sol. & Insol. & Sol. \\
\hline 1 & UV & 10 & 16 & 61 & 67 & 4.0 & 85 & 83 \\
\hline 2 & UV & 25 & 35 & 45 & 70 & 4.2 & 83 & 80 \\
\hline 3 & UV & 120 & 55 & 21 & 89 & 6.7 & 75 & 73 \\
\hline 4 & AIBN & 20 & 48 & 26 & 67 & 4.2 & 81 & 85 \\
\hline 5 & None & 20 & 27 & 44 & 58 & 3.4 & 85 & 87 \\
\hline
\end{tabular}
No. 1).

Table I. Effect of initiation mode and polymerization time on the polymerization of benzenedithiol to diethynylanthracene in THF at $50^{\circ} \mathrm{C}$ under nitrogen atmosphere ${ }^{\mathrm{a}}$

a $[\mathrm{BDT}]_{0}=[\mathrm{DEA}]_{0}=0.085 \mathrm{moll}^{-1}$.

b None: in dark; $[\mathrm{AIBN}]_{0}=0.0016 \mathrm{moll}^{-1}$; UV: High pressure mercury lamp, $450 \mathrm{~W}$.

c Copper acetylide method.

d IR method. 
be due to the presence of a small amount of oxygen remaining in the reaction solution. The THF solvent was treated previously with $\mathrm{NaOH}$ and then refluxed over sodium wire for one or two days and distilled under nitrogen atmosphere before use. Therefore, the existence of a peroxide may be neglected. Regardless of the initiation mode, the obtained insoluble and soluble polymers are abundant in the cis vinylene content, reaching above $80 \%$ except for No. 3 in Table I.

Figure 3 shows the effect of polymerization time on the microstructure and the numberaverage molecular weight $\left(\bar{M}_{n}\right)$ of the polymers. The molecular weight of the insoluble and the soluble polymers increased gradually with the polymerization time. This behavior indicates that polymerization progresses stepwise in a similar manner to a condensation polymerization. On the other hand, both the insoluble and soluble polymers maintain high cis contents in the beginning of the polymerization, and then gradually decrease with polymerization time. This means that the cis vinylene in the polymer backbone isomerizes easily to the trans one by UV irradiation with the proceeding of the polymerization,

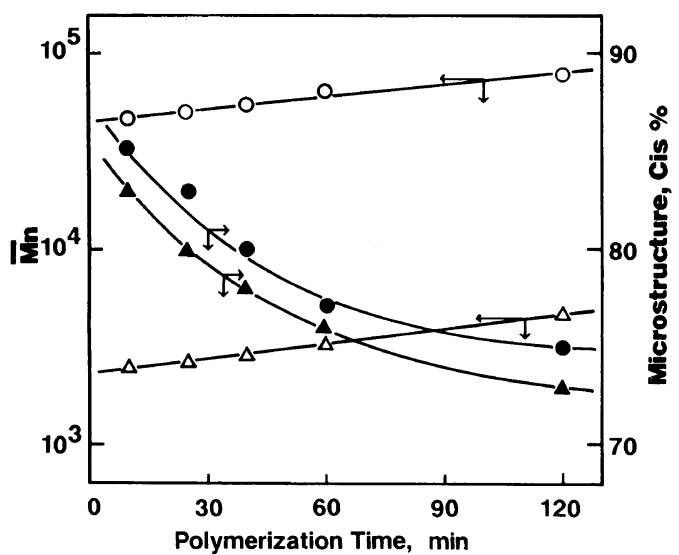

Figure 3. Relation between polymerization time and $\bar{M}_{n}$ and the microstructure of the polymers polymerized in $\mathrm{THF}$ at $50^{\circ} \mathrm{C}$ by $\mathrm{UV}$ irradiation under nitrogen atmosphere: $[\mathrm{BDT}]_{0}=[\mathrm{DEA}]_{0}=0.085 \mathrm{moll}^{-1} ; \bigcirc$ and indicate the insoluble polymer; $\triangle$ and $\boldsymbol{\Delta}$ show the soluble polymer. or the configuration of the polymer growing end would be favorable to connect with reactive end groups of monomers and oligomers in the trans from. These problems will be discussed later on.

\section{Isomerization of Polymers by UV Irradiation}

As shown in Figure 3, the cis content of the polymer decreases with increasing polymerization time. It seems that the vinylene unit in the polymer chain undergoes isomerization from the cis configuration to the trans one, that is, the polymer chain may adopt a more energetically favorable configuration in which the vinylene unit in the polymer chain would take the trans configuration. To confirm this, the resulting insoluble polymer (a point at $120 \mathrm{~min}$. in Figure 3) was isolated and used to make a $\mathrm{KBr}$ disk, which is directly illuminated by UV light. As shown in Figure 4, even in the solid state, the vinylene unit in the polymer backbone isomerized from the cis configuration to the trans one. About $30 \%$ of the cis

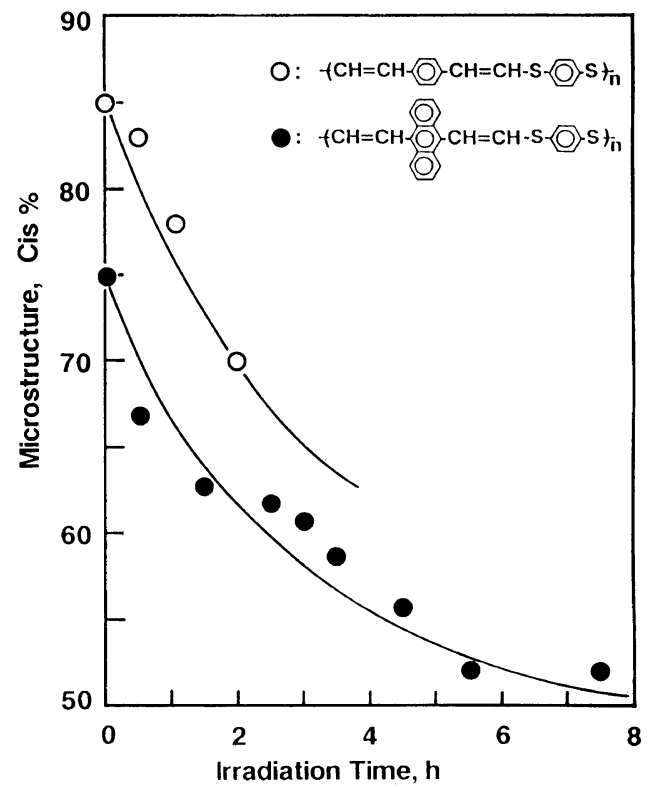

Figure 4. Isomerization behavior of insoluble polymers at $30^{\circ} \mathrm{C}$ : $\bigcirc$, by UV irradiation under air; $\bigcirc$, by visible light at $539.6 \mathrm{~nm}$ under nitrogen atmosphere $(\mathrm{KBr} \text {, disk })^{4}$ 
vinylene compared to the starting state isomerized to the trans one after $5 \mathrm{~h}$. This explains that the polymer composed of BDT with DEA easily isomerizes from the cis to the trans, when a sufficient excitation energy is afforded by UV irradiation. The cis-to-trans isomerization occurred quickly at the beginning, and then turned smooth. The cis vinylene content decreased to $52 \%$ after about $8 \mathrm{~h}$. The restriction of the polymer conformation, the interaction to the matrix and the decrease in a transparency of $\mathrm{KBr}$ disk may interfere with fast isomerization.

Like the polymer composed of BDT with DEA, the polymer of BDT and 1,4-diethynylbenzene can also isomerize from the cis configuration to the trans one. ${ }^{4}$ Within $2 \mathrm{~h}$, the cis content decreased from $85 \%$ to $70 \%$ by the irradiation of visible light $(539.6 \mathrm{~nm}) .{ }^{4}$ In this polymer, another interesting feature is that the crystallinity of the polymer obtained in solid state polymerization also decreases with decreasing the cis vinylene content in the polymer backbone. It seems that the isomerization of microstructure of the polymer would induce the reconstruction of the polymer crystalline phase.

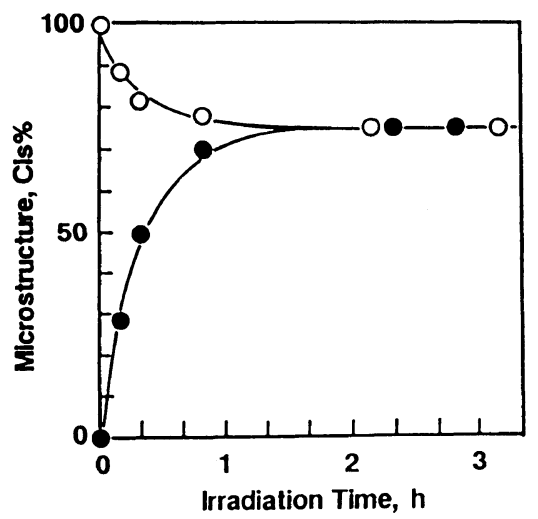

(A)
Isomerization of Model Compound by UV Irradiation

The isomerization of model compound, 9,10-bis(phenylthiovinylene)anthracene by UV irradiation or in the presence of a radical source was also studied. The $c i s$ isomer of the model compound in a pyrex tube equipped with a three-way stopcock was irradiated in THF by UV light at $25^{\circ} \mathrm{C}$ under nitrogen atmosphere. After a certain period, a definite fraction was taken out from the reaction tube, and then, the solvent was removed by evaporation under reduced pressure. Successively, it is dried in vacuo at room temperature and used to determine the cis content by the IR method.

As shown in Figure 5B, the cis isomer was converted quickly into the trans one by UV irradiation, and the isomerization proceeded completely after $3 \mathrm{~h}$. On the other hand, the resulting trans isomer did not come back to the cis one under the same conditions. This behavior is different from the UV-induced isomerization of $\beta$-phenylthiostyrene (Figure 5A), in which the isomerization of the cis and trans isomer was carried out in hexane at $30^{\circ} \mathrm{C}$ under nitrogen atmosphere by UV irradiation, respectively. Both the cis and the trans isomers

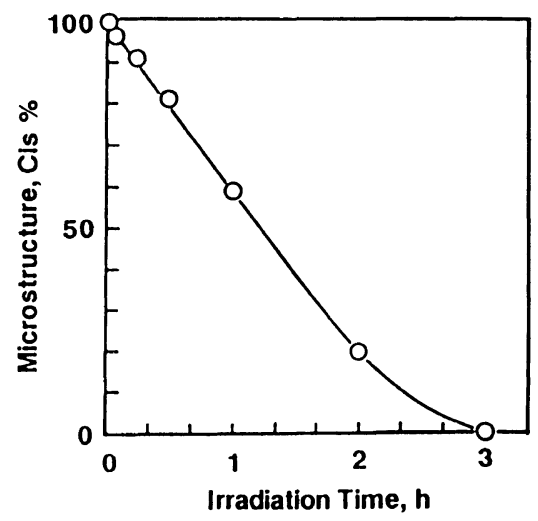

(B)

Figure 5. Isomerization of model compounds by UV irradiation: (A) $\beta$-phenylthiostyrene in hexane at $30^{\circ} \mathrm{C}$ under nitrogen atmosphere, $[\text { cis }]_{0}=[\text { trans }]_{0}=0.67$ moll ${ }^{-1}$ (NMR method). ${ }^{11}$ (B) 9,10-bis(phenylthiovinylene)anthracene in THF at $25^{\circ} \mathrm{C}$ under nitrogen atmosphere, $[\text { cis }]_{0}=0.026 \mathrm{moll}^{-1}$ (IR method). 


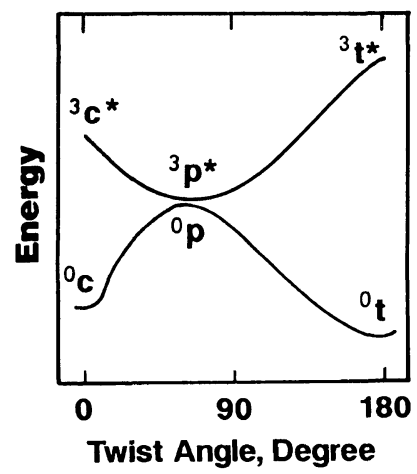

(A)

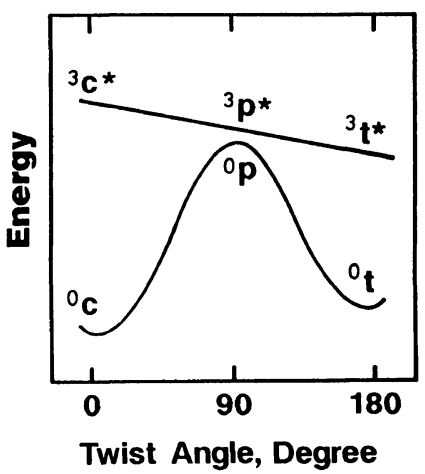

(B)

Figure 6. Potential energy surface for isomerization of vinylene bond by UV irradiation: (A) $\beta$ phenylthiostyrene, ${ }^{11}$ calculated by MINDO/3. (B) 9,10-bis(phenylthiovinylene)anthracene expected based on the experimental results.

could undergo isomerization to the opposite configuration, and reached a photostationary state of the cis $75 \%$ and the trans $25 \%$ after $2 \mathrm{~h} .{ }^{11}$ Similarly, such characteristic isomerization behavior was also observed in the photoisomerization of stilbene and $\beta$-anthrylethylene type compounds. ${ }^{12}$ The former attained a photostationary state and the latter gave only the trans isomer.

This behavior can be accounted for on the basis of the energy levels of the transition states for the isomerization. Figure 6 shows the relationship between the potential energy surfaces of the compounds and the twist angle around their vinylene bond.

In the case of $\beta$-phenylthiostyrene (Fig. 6A), UV-induced excitations of cis and trans isomers give cis triplets $\left({ }^{3} c^{*}\right)$ and trans triplets $\left({ }^{3} t^{*}\right)$, respectively. However, neither ${ }^{3} c^{*}$ nor ${ }^{3} t^{*}$ is stable and both readily twist around their vinylene bond into the most stable triplet, an almost perpendicularly twisted triplet $\left({ }^{3} p^{*}\right)$ shown in Figure 6A. ${ }^{3} p^{*}$ is very close in energy to ${ }^{0} p$ and undergoes deactivation to ${ }^{0} \alpha$ through intersystem crossing. ${ }^{12-14}$ Then, the resulting ${ }^{0} p$ collapses to either cis or trans isomers in the ground state at a certain ratio. Therefore, after irradiation for a sufficient time, the reaction results in a photostationary state comprising a certain ratio of both isomers. The cis isomer content is more than the trans one in the photostationary state, because the ${ }^{3} p^{*}$ lies close to the cis side. Figure 6A coincides with both the experimental results and quantum chemical calculations. ${ }^{11,15}$

On the other hand, in the case of 9,10bis(phenylthiovinylene)anthracene, the cis isomer was irreversibly isomerized to the trans isomer, since no reverse reaction from their trans to cis took place by the photoisomerization. It is considered that there is no participation of ${ }^{3} p^{*}$ as an important intermediate, since it would give a mixture of both isomers. The authors have proposed a potential energy diagram depicted in Figure $6 \mathrm{~B}$ based on the experimental results. Thus, ${ }^{3} c^{*}$ resulting from excitation undergoes twisting around the vinylene bond to attain ${ }^{3} t^{*}$ by passing through a perpendicular conformation. The excited state does not stay at the ${ }^{3} p^{*}$ conformation for a sufficient lifetime to be deactivated to the ground state, and this conformation would be only a simple point to be quickly passed. ${ }^{13,14}$ Therefore, the excited state would undergo deactivation from ${ }^{3} t^{*}$ to the ground state, instead of the decay from ${ }^{3} p^{*}$ to the ground state. On recycling these processes, the irreversible isomerization takes place successively. 


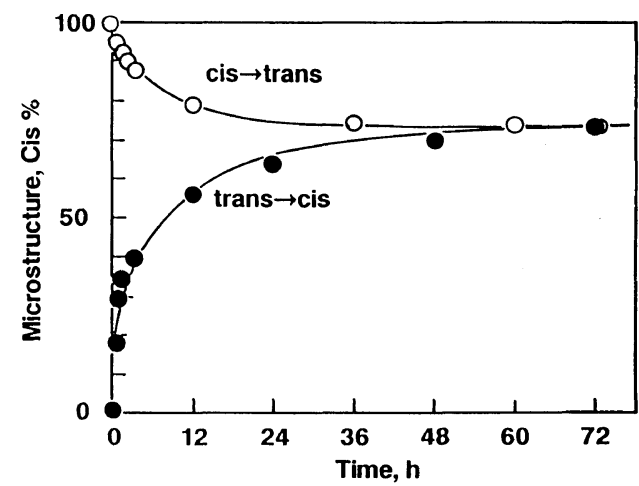

Figure 7. Isomerization of 9,10-bis(phenylthiovinylene)anthracene in the presence of thiophenol in THF at $50^{\circ} \mathrm{C}$ under nitrogen atmosphere: $[\text { cis }]_{0}=[\text { trans }]_{0}=0.026$ moll ${ }^{-1}$; [thiophenol $]_{0}=0.00026 \mathrm{moll}^{-1}$ (IR method).

This dramatic feature of the isomerization of the model compound containing anthracene is considered due to the highly conjugated $\pi$ system of anthracene linked to the vinylene unit.

\section{Isomerization of Model Compound in the} Presence of Radical

The model compound containing anthracene ring isomerizes easily from its $\mathrm{cis}$ isomer to the trans one in irreversible isomerization by UV irradiation just mentioned above. How does it occur in the presence of a radical source, such as thiophenol in the THF solution? In the presence of a small amount of thiophenol in a THF solution of the model compound, both the cis and trans isomers can undergo isomerization to the opposite configuration gradually. After reaction for a sufficient time, the reaction reaches an equilibrium state comprising a certain ratio of both isomers, in which the cis isomer contains $74 \%$, much more than the trans one (Figure 7).

This thermal isomerization behavior compared to the UV-induced isomerization suggests that the thiol in the reaction solution acts as a catalyst to stimulate the isomerization on heating. ${ }^{16}$ This indicates that thiyl radicals are the active catalytic agents whose reversible addition and elimination to and from the

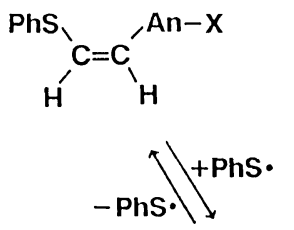<smiles>[X][GaH]C=C([SbH])[Hg]</smiles>

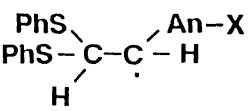

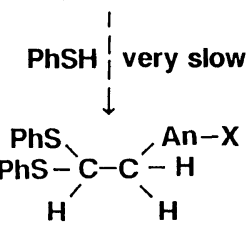

Scheme 3. Mechanism of isomerization in the presence of thiophenol.

vinylene unit may result in isomerization as shown in Scheme 3, where An-X means phenylthiovinyleneanthryl.

The absence of the methine and methylene absorption peaks at 2800 to $3000 \mathrm{~cm}^{-1}$ and other peaks different from the starting substrates and ${ }^{1} \mathrm{H}$ NMR spectra demonstrate that the catalysis of the isomerization by thiophenol could not bring about a saturated adduct formation. Even though the adduct is formed, the amount of it would be negligibly small. The reason would be due to the high degree of stability, i.e., low reactivity of the intermediate carbon radical, in which the adjacent anthracene group stabilizes the methine radical to prevent the abstraction of the hydrogen from thiophenol forming a saturated adduct. Steric factors of the intermediate carbon radical may also affect the low rate of hydrogen abstraction from the thiol.

\section{REFERENCES}

1. E. Kobayashi, T. Ohashi, and J. Furukawa, Makromol. Chem., 189, 2525 (1986).

2. E. Kobayashi, T. Ohashi, and J. Furukawa, J. Polym. Sci., Polym. Chem. Ed., 25, 2077 (1987).

3. O. Nuyken and F. Siebzehnrübl, Polym. Bull., 19, 371 (1987)

4. E. Kobayashi, T. Ohashi, T. Yagi, and J. Furukawa, 


\section{J. JIANG et al.}

Rep. Jpn. Synthetic Fiber Institute, 44, 95 (1987).

5. E. Kobayashi, J. Jiang, and J. Furukawa, Polym. J., 22, 266 (1990).

6. E. Kobayashi, J. Jiang, H. Ohta, and J. Furukawa, J. Polym. Sci., Polym. Chem. Ed., in press.

7. D. S. Chemla and J. Zyss, "Nonlinear Optical Properties of Organic Molecules and Crystals," Academic Press, New York, N. Y., 1987.

8. D. J. Williams, "Nonlinear Optical Properties of Organic and Polymeric Materials," ACS Symposium Series No. 233, The American Chemical Society, Washington, DC, 1983.

9. E. Kobayashi, T. Ohashi, and J. Furukawa, Polym. J., 21, 111 (1989).

10. D. Morero, A. Santambrogio, L. Porri, and F. Ciampelli, Chem. Ind., 41, 758 (1959).
11. E. Kobayashi, H. Takayanagi, S. Aoshima, and J. Furukawa, Polym. Prepr. Jpn., 37, 1511 (1988).

12. T. Arai, T. Karatsu, H. Misawa, Y. Kuriyama, H. Okamoto, T. Hiresaki, H. Furuuchi, H. Zeng, H. Sakuragi, and K. Tokumaru, Pure Appl. Chem., 60, 989 (1988).

13. K. Tokumaru, Gendai Kagaku, 6, 16 (1988), and reference cited therein.

14. T. Arai and K. Tokumaru, J. Synth. Org. Chem. Jpn., 44, 999 (1986).

15. H. Takayanagi, M. Sc. thesis, Science University of Tokyo, Noda, 1989.

16. A. A. Oswald, K. Griesbaum, B. E. Hundson, Jr., and J. M. Bregman, J. Am. Chem. Soc., 86, 2877 (1964). 